\title{
Periangular transmasseteric infraparotid approach in the treatment of condylar-base and low condylar-neck fractures
}

\author{
Hirjak $\mathrm{D}^{1}$, Vavro $\mathrm{M}^{1}$, Dvoranova $\mathrm{B}^{1}$, Galis $\mathrm{B}^{1}$, Simko $\mathrm{K}^{1}$, Malicek $\mathrm{L}^{2}$, Machon $\mathrm{V}^{3}$, Neff $\mathrm{A}^{4}$ \\ Department of Oral and Maxillofacial Surgery, Comenius University, University Hospital Ruzinov, \\ Bratislava, Slovakia. hirjak.dusan@gmail.com
}

\begin{abstract}
AIM: Mandibular condylar fractures account for 25 to $52 \%$ of all mandibular fractures. Though current literature favors open reduction and internal fixation (ORIF) of condylar-base and low condylar-neck fractures, extraoral approaches are usually considered to be complicated by the risk of facial nerve injury and other possible complications. This study was undertaken to demonstrate that the periangular transmasseteric infraparotid surgical approach (TMIP) to condylar-base and low condylar-neck fractures provides excellent access to the bony fragments with minimal risk of complications such as facial nerve and parotid gland injury. PATIENTS: In the period from January 2010 to December 2018, 81patients (96 fractures) with condylar-base and low condylar-neck fractures underwent ORIF via periangular transmasseteric infraparotid surgical approach.

RESULTS: The results of this retrospective study showed minimal postoperative complications. The periangular transmasseteric infraparotid surgical approach allowed precise anatomic repositioning and fixation of the bony fragments in almost all cases except for two juvenile cases with noticeable scars and one case with plate fracture. There were no transient or permanent facial nerve palsies, parotid gland or salivary fistulae complications during a 12-month follow-up period.

CONCLUSION: The periangular infraparotid transmasseteric approach to ORIF of condylar-base and low condylar-neck fractures is an effective and safe approach allowing accurate anatomic reposition and fixation of the fragments with minimum surgical complications (Tab. 1, Fig. 12, Ref. 21). Text in PDF www.elis.sk KEYWORDS: condylar base, condylar neck, open reduction and internal fixation (ORIF), facial nerve injury (FNI), transient/permanent facial nerve palsy, parotid gland injury.
\end{abstract}

\section{Introduction}

Mandibular condylar fractures (MCFs) account for 25 to $52 \%$ of all mandibular fractures $(1,2,3,4)$. The management of MCFs remains controversial in the literature. Because of its less invasive nature, the conservative treatment using closed reduction used to be favored for decades. However, long-term complications such as malocclusion, pain, loss of vertical height and temporomandibular disorders (TMD) were often the sequelae requiring secondary surgical correction. Recently, the literature supports the view that treatment outcomes utilizing open reduction and internal fixation (ORIF) are superior to conservative treatment using closed reduction. Along with the development of improved surgical techniques and internal fixation materials, ORIF has be-

${ }^{1}$ Department of Oral and Maxillofacial Surgery, Comenius University, University Hospital Ruzinov, Bratislava, Slovakia, ${ }^{2}$ Department of Stomatology and Maxillofacial Surgery, Comenius University, OUSA Hospital, Bratislava, Slovakia, ${ }^{3}$ Department of Oral and Maxillofacial Surgery, University Hospital of Charles University, Prague, Czech Republic, and ${ }^{4}$ Department of Oral and Maxillofacial Surgery, University Hospital of Marburg, Faculty of Medicine, Philipps University, Marburg, Germany

Address for correspondence: D. Hirjak, MD, Department of Oral and Maxillofacial Surgery, Comenius University, University Hospital Ružinov, Bratislava, Slovakia. come widely accepted and applied $(1,3,4,5,6,7)$. One of the most practical classification systems of MCFs was proposed by Loukota et al $(2,5,8)$ and was recently modified by Neff et al $(8,9)$. This classification divides the fractures by landmark-based fracture line location occurring in the base, neck and head of the condylar process $(2,9)$.

Condylar fractures differ markedly from other mandibular fractures with respect to the anatomy of the surrounding tissues. Therefore, MCFs are more easily managed via an external approach, or intraoral endoscopically assisted approach. Extraoral approaches are complicated by the necessity to avoid facial nerve injury (FNI) which may result in transient facial nerve injury (TFNI) and/or permanent facial nerve injury (PFNI) $(1,2)$. Other complications that can be associated with ORIF of condylar fractures are intraoperative hemorrhage, parotid gland injury and/or postoperative infection, Frey syndrome, salivary fistula, and/or scarring $(7,10)$.

The International Bone Research Association (IBRA), Condylar Fracture Symposium 2012 in Marseille (8) pointed out that ORIF may by now be considered as a treatment of choice for both displaced and dislocated condylar base and condylar-neck fractures: The selection of the ideal surgical approach for condylar-base and low condylar-neck fractures, however, is still debated, with percutaneous procedures being the most preferred surgical approaches. 
Thus, according to the 2012 IBRA Marseille consensus conference survey, the retromandibular followed by the high submandibular and periangular approaches were considered preferred ways of surgical access to condylar-base and condylar neck-level fractures $(1,2,8)$, whereas the transoral endoscopically assisted approach, based on the authors' experience and supported by the literature, is recommended for selected, "easy" cases such as lower condylar base fractures with lateral overlap $(5,11,12)$.

According to the literature, the so called "retromandibular" (i.e., deep retroparotid, trans- and anteroparotid) and "submandibular" (i.e., Risdon approach, low and high submandibular approaches, high cervical approach) are the most frequently recommended percutaneous approaches. Basically, these surgical approaches differ because of their relationship to the parotid gland (retro- viz. subparotid, transparotid, anteroparotid and infraparotid) and the masseter muscle, using either a transmasseteric incision (antero- and transparotid) or partial dissection (retroparotid), or a full or subtotal transsection of the masseteric muscle, the latter utilizing an angular/high submandibular approach which gained popularity during the last decade because of lower occurrences of both TFNI and PFNI $(3,4,8,10,12,13,14,15,16,17,18)$.

The aim of this retrospective study was to present the authors " experience with the periangular transcutaneous (transplatysmal, infraparotid, transmasseteric) surgical approach to MCFs and to evaluate the outcomes after treatment of condylar-base (CBFs) and low condylar-neck fractures (CLNFs).

\section{Patients and methods}

Between January 2010 and December 2018, at the Department of Oral and Maxillofacial Surgery, University Hospital Ruzinov
Tab. 1. Results of ORIF of CBFs and CNFs in details.

\begin{tabular}{|c|c|c|c|c|c|c|}
\hline Age (years) & up to 15 & $16-25$ & $26-35$ & $36-45$ & $46-55$ & $56-$ \\
\hline patients & 2 & 20 & 28 & 17 & 9 & 15 \\
\hline Cause of the fracture & violence & sport & \multicolumn{2}{|c|}{ work } & raffic & falls \\
\hline patients & 21 & 12 & \multicolumn{2}{|c|}{7} & 12 & 29 \\
\hline Localization of the fracture & unilatera & ilateral fr. & \multicolumn{4}{|c|}{ single/comb. with 1 or more mand.fr. } \\
\hline patients & \multicolumn{2}{|c|}{$66 / 15$} & \multicolumn{4}{|c|}{$30 / 51$} \\
\hline Fragments position & \multicolumn{2}{|c|}{ lateral displacement } & \multicolumn{2}{|c|}{ medial condyle dislocation } & \multicolumn{2}{|c|}{ medial displacement } \\
\hline patients & \multicolumn{2}{|c|}{57} & \multicolumn{2}{|c|}{20} & \multicolumn{2}{|r|}{4} \\
\hline Type of fixation & \multicolumn{2}{|c|}{1 straight pl. } & traight pl. & lambda & \multicolumn{2}{|c|}{ trapezoid } \\
\hline patients & \multicolumn{2}{|c|}{14} & 22 & 31 & \multicolumn{2}{|c|}{14} \\
\hline
\end{tabular}

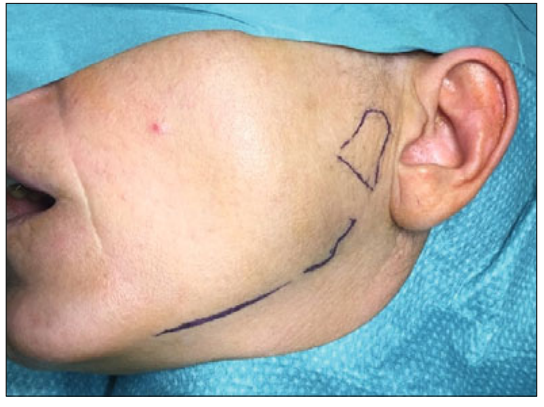

Fig. 1. Main anatomic landmarks and the fracture line, a 30-40-mm long curved skin incision is marked around the palpable mandibular angle.

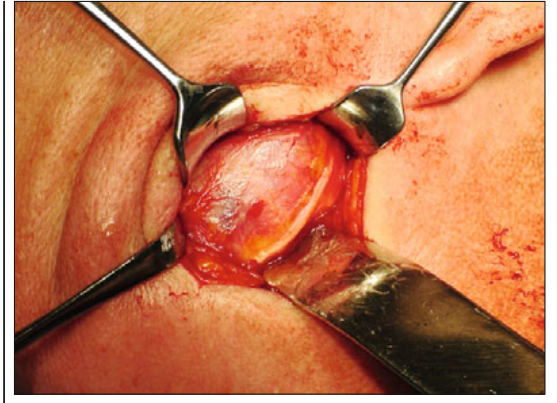

Fig. 2. M. masseter exposed, facial nerve's marginal branch. in Bratislava, 81 patients underwent ORIF of CBFs and CLNFs, utilizing the periangular infraparotid transmasseteric surgical approach. Most of the patients were operated by the first and second authors. According to the 2005 Loukota classification, 78 patients were classified as CBFs and 13 as CLNFs. Mean age was 46 years, ranging from 14 to 69 years; 24 patients were female and 57 were male. There were 66 unilateral and 15 bilateral fractures. In 51 patients, the condylar fracture was associated with other mandibular fractures. Inclusion criteria for ORIF were: 1) patients older than 12 years, 2) trauma-related malocclusion (lateral/frontal open bite), 3) displacement of the condylar fragment with ramus shortening, dislocation of the condyle out of the fossa, medially displaced con-

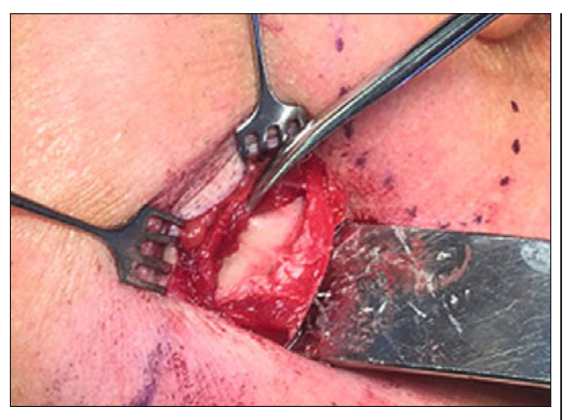

Fig. 3. The muscle dissection was made directly to the bone and the masseteric muscle was dissected upward.

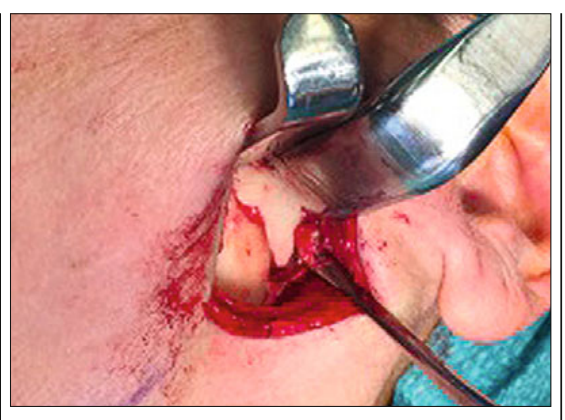

Fig. 4. Exposed shorten ramus and proximal fragment.

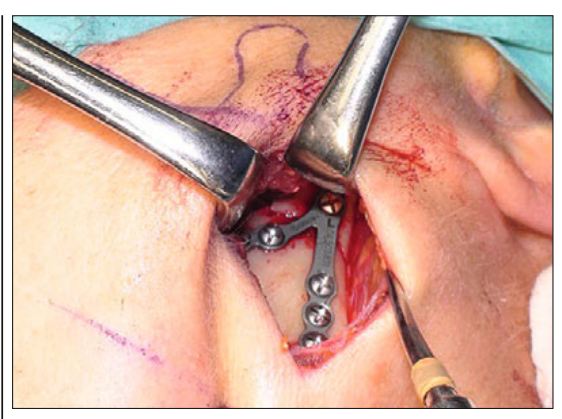

Fig. 5. ORIF using lambda plate. 


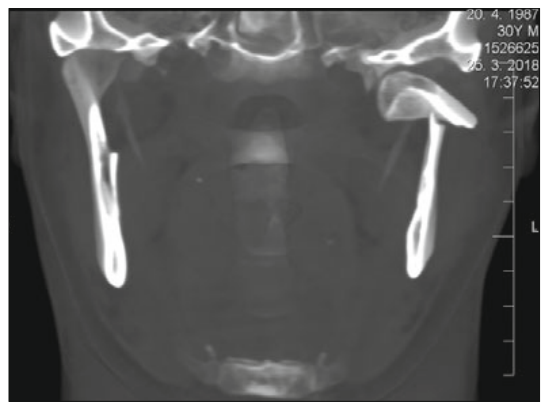

Fig. 6. CT scan, coronal view of displaced and dislocated fragment.
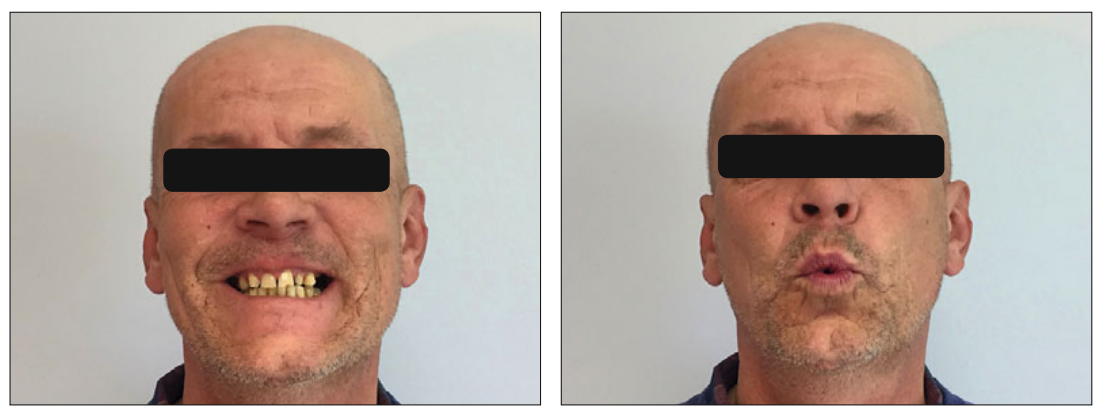

Figs 8 and 9. Facial nerve function 7 days after ORIF.
(17) and Meyer/Wilk (6). After outlining the main anatomic landmarks and the fracture line, a $30-40 \mathrm{~mm}$ long curved skin incision was marked and made around the palpable mandibular angle (Fig. 1). Below the skin and subcutaneous tissue, the platysma muscle was identified. For better visibility, the wound can be widened by subcutaneous undermining in all directions. The masseter muscle (MM) was exposed and widely undermined superiorly and posteriorly, which allows the surgeon to visually identify the facial nerve branches (FNB). In most of cases the marginal branch traverses the lower angular border, while some thinner buccal branches run 3-5mm cranially (Fig. 2). There are cases where facial nerve branches are not visible.

The muscle dissection was made above the visible marginal nerve branch directly to the bone and the masseteric muscle was dissected upward to expose the ramus up to the fracture line (Fig. 3). It is important to release the masseter muscle from the posterior border and to perform muscle transsecdylar fragment. Exclusion criteria were: 1) patients younger than 12 years, 2) edentulous alveolar ridges, 3) high risk of general anesthesia, 4) noncompliant patients.

The position of the fragments was determined from an orthopantomogram (OPG) and/or CT scans. The type of osteosynthesis was selected according to the location of the fracture and dimensions of the fragments (Tab. 1).

Surgical procedures were performed under general anesthesia via nasotracheal intubation. A perioperative intermaxilary fixation (IMF) was applied using intermaxillary screws and wire. Active postoperative functional rehabilitation was employed using guiding elastics applied for 2-3 weeks. A liquid-to-soft diet was maintained for 6 weeks postoperatively.

\section{Surgical technique}

The periangular infraparotid transmasseteric surgical approach used in this study followed the technique described by Rasse/Eckelt tion, i.e. allowing for a full release of the muscle from the portion which remains fixed on the mandibular angle. The fracture stumps were then identified and mobilized from the soft tissue (Fig. 4).

After gentle cranial (proximal fragment) and caudal traction (distal fragment) with retractors, the surgeon will have a good view of the fragments. After anatomic reduction, the fractured stumps were rigidly fixed using different plates (2 straight plates, lambda and trapezoid plates-TCP, chosen according to the individual fracture type) and appropriate screws (Fig. 5). In complex low-neck fractures cases, the authors will typically first fix the plate to the proximal fragment with 2 screws, then reduce the fracture. The posterior border of the ramus and the sigmoid notch are the most important landmarks for proper reduction. After checking the position of fragments in both of these areas, screws were fixed to the distal fragment. In pediatric patients and some low-neck fractures, there is very little space for 2 plates when approached via the periangular approach. In these cases, the authors prefer lambda or TCP plates (Figs 6 and 7).
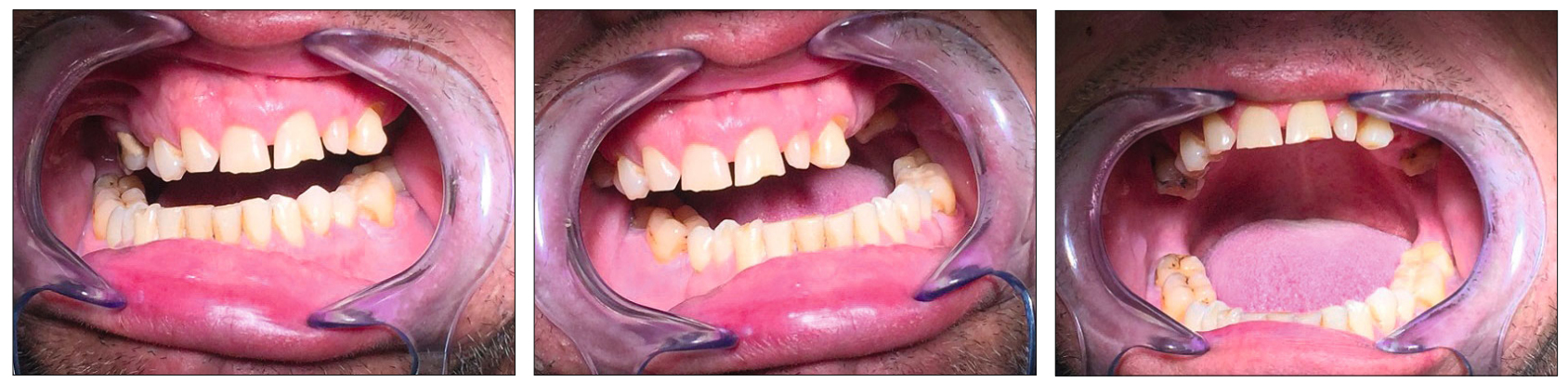

Figs 10, 11 and 12. Mandibular function 1 month after ORIF. 


\section{Results}

The patients were seen at follow-ups after 7 days, 1, 3, 6 and 12 months. Postoperatively, the following parameters were assessed: occlusion, maximal interincisal opening (MIO), deviation of the mandible during function, facial nerve function (FNF), occurrence of any salivary fistulae and the aesthetic result (scar) (Figs 8, 9, 10, 11 and 12).

At 3 months, postoperatively, no malocclusions were observed and the mandibular function was determined to be good (Tab. 1, postoperative MIO). There were no FNI resulting in transient and/ or permanent functional impairment. No complications connected with injury to the parotid gland (Frey's syndrome, salivary fistula, etc.) were observed. In 2 patients with single straight plates, the plates have fractured. Surgical scarring was considered acceptable in most patients. However, 2 patients, aged 14-16 years healed with wide aesthetically displeasing scars. The details of postoperative results are depicted in Table 1.

\section{Discussion}

The treatment of condylar fractures should be based on high level of evidence. The development of functionally stable fixation allows for open reduction and biomechanically stable fixation of condylar fractures without the need of postoperative MMF (6, 9, 13, 17).

In the conclusions of IBRA Condylar Fracture Osteosynthesis Symposium 2012 in Marseille, Neff et al reported it was the consensus of the attendees that ORIF may be considered as the treatment of choice for both displaced and dislocated condylar base and neck fractures (8).

Choosing an appropriate approach for surgical treatment of mandibular condylar-base fractures (MCBFs) is paramount, yet controversial. The main concerns in managing condylar fractures are adequacy of surgical exposure, correct three-dimensional alignment of bone fragments and stable internal fixation, which are mutually interdependent in order to assure an anatomically correct and stable result from both biomechanical and functional standpoints.

Futher, a corresponding goal is to minimize the risk of facial nerve injury (FNI) resulting in transient (TFNP) and/or permanent facial nerve palsy (PFNP). While initially aesthetically concerning, TFNPs are typically reversible within a 3-6-month range. By contrast, PFNP must be considered as a major complication to be avoided by employing both proper surgical technique and skillful soft tissue management. These neural concerns, as well as complications associated with the parotid gland involve a selection of appropriate surgical approaches and osteosynthesis systems.

Recently, the data on FNI in relation to surgical approaches during condylar fracture treatment have been reported $(1,2)$. These studies identified approaches associated with minimum risk for the facial nerve. However, high FNI rates, including PFNP, continue to be reported $(7,19,20)$. This may be attributed to either lack of surgical experience and/or selection of inappropriate surgical approach such as the use of the preauricular approach for base fractures. According to the literature, the most preferred and most frequently published approaches for ORIF of condylar-base and low condylar-neck fractures are still "submandibular" (i.e. low submandibular), preauricular and "retromandibular" (i.e. retroparotid deep subparotid retromandibular approach), in contrast to the more recent approaches such as the antero- and transsparotid approaches, and the angular/periangular/high submandibular approach. The latter represents an approach with the lowest complication rate compared to all others cited $(3,4,10,12,13$, $14,15,16,17,18)$.

Both the anteroparotid and transparotid approaches commence with a retromandibular incision, sometimes extended to the lower preauricular region (so-called, lazy S“) (5). Both antero- and transparotideal approaches exhibit a significantly higher FNP risk, especially TFNPs, as compared to the angular approach $(7,19,20)$. The transparotid approach has the following advantages: the incision is closest to the condylar process, it creates an acceptable scar and gives direct and orthogonal exposure to the fractured fragments, which is an advantage with fractures of the condylar neck region closer to the condylar head region. Also, the transparotid approach is touted to provide better access in the treatment of fractures located in the middle part of the neck as it offers a better access to the posterior border of the mandible allowing a placement of two plates in cases which typically are less amenable to double plating when using the anteroparotid and/or angular approach. Still, it must be remembered that this advantage is at the cost of a significantly higher rate of FNI as compared to the angular approach. When a transparotid dissection is performed, a deliberate identification of the facial nerve is associated with a significantly higher rate of FNI, as compared with approaches that do not dissected through parotid tissue $(19,20)$. However, the rates of FNI described for the transparotid approach, in case the nerve is not deliberately exposed, are almost equal to those of the anteroparotid approach (19). Further, the use of the transparotid approach in inexperienced hands can create several serious complications such as salivary fistula (sialocele).

Guerrissi et al (2002) and Wilson et al (2005) (11, 20), utilizing the retromandibular and transparotid approach presented facial nerve complications up to $30 \%$. Li Z et al (14) published their results after using a modified tragus edge approach (MTEA; viz. lazy-S incision) for mid-level or low condylar fractures. The occurrence of facial nerve dysfunction after MTEA as compared with retromandibular transparotid approaches was $3.4 \%$ and $10.9 \%$, respectively while parotid fistula was present in $0.0 \%$ and $6.3 \%$, respectively. This is basically in line with the metaanalysis by $\mathrm{Al}$ Moraissi et al $(1,2)$, who reported an average rate of TFNIs between $4.7 \%$ (no exposure of the facial nerve) and $7.9 \%$ (accidental exposure of the facial nerve). Al Moraissi, Ellis, Neff compared to $4.6-6.3 \%$ for the anteroparotid approach $(1,2$, 8). In striking contrast though, the rate of TFNPs for the angular approach was $\geq 0.9 \%$. To date, no PFNIs have been reported for the angular approach, which also applies for the anteroparotid approaches $(1,2)$. 
184-189

Mauro Pau et al (17) published their experience using a modified high submandibular (i.e. angular) approach for condylar-base fractures. The main advantages reported were low risk of facial nerve injury, avoidance of parotid gland injury and good aesthetic results. These authors concluded that this approach allows the surgeon to easily insert the plates with minimal traction on the soft tissues. The authors also stated that the access to the bony fragments through the masseter, rather than through the parotid gland, reduces the risk of parotid gland injury. As described by Rasse/Eckelt/Meyer/Wilk $(6,13)$, the masseter is released at the level of an anatomically nerve-free window between the buccal and marginal mandibular branches of the facial nerve, thereby avoiding damage to that nerve.

However, it should be stressed that the high submandibular approach (i.e., the angular and periangular approach) is also potentially misleading when touted as "anteroparotid transmasseteric approach“ (cf. e.g., Al Moraissi, Louvrier et al, 2018) (1,2) and as such has been portrayed as potentially the safest way to approach the condylar-base and low condylar-neck fractures. The "anteroparotid transmassteric" (i.e., utilizing full or at least subtotal transsection of the masseter muscle) approach should not be confused with the anteroparotid approach, which involves a limited incision into the masseteric muscle as in the transparotid approach. Therefore, to avoid confusion the transmasseteric anteroparotid approach should be more precisely termed the transmasseteric infraparotid approach (TMIP).

According to the meta-analysis by Al-Moraissi, Louvrier et al $(1,2)$ showing that the periangular/angular/perimandibular/high submandibular approach ( viz. "transmasseteric infraparotid approach“, TMIP) is associated with lowest rate of TFNI (0-0.9\%) and permanent facial nerve injury (PFNI; $0 \%$ ) comparing to other more popular approaches, the authors explained these results using the following reasoning: in most cases, direct visualization of the marginal mandibular branch of the facial nerve allows the surgeon direct visualizations of the bony fragments and orthogonal application of the plate(s) and screws, and the infraparotid approach avoids injury of the parotid gland. Further, because the TMIP approach employs the nerve-free window between the buccal branches and the marginal mandibular branches, it reduces the risk of FNI. Since the parotid capsule is not penetrated, the injury to the parotid gland is also minimized. A curved skin incision around the angle or parallel to the palpable mandibular angle as described by Meyer et al (15) shortens the distance between skin incision and bony fragments thus decreasing the traction on the associated soft tissues and creating a minimally visible periangular scar.

The extraoral, transcutaneous peri-angular approach has gained some popularity during the last decade $(1,2,6,7,13,15,19,20$, 21). Despite the excellent results reported with regard to low incidences of FNI and injury of the parotid gland, the periangular approach is not well represented in the literature. From the risk assessment standpoint, the periangular transmasseteric infraparotid approach to the surgical management of condylar-base and low condylar-neck fractures is well suited as a replacement for the traditional approaches associated with the high risk of PFNIs. The periangular skin incision is simple and can also be used in obese patients. The main advantages lie in the direct visualization of the marginal mandibular branch of the facial nerve in most cases, which is bypassed from above while avoiding traction on the nerve. The transmasseteric approach with its (sub)total transection of the masseter muscle reduces the hook traction and allows for direct visualizations of the fragments and easy placement of the plates, although the application of the screws becomes more oblique the higher the fracture is located. Furthermore, the infraparotid transmasseteric approach allows the surgeon to avoid injury of the parotid gland.

\section{Conclusion}

The results of the present retrospective study demonstrate that the periangular infraparotid transmasseteric approach is an effective and safe approach for ORIF of condylar-base and low condylar-neck fractures $(1,6,7,13,19,20)$. As there is an abundance of confusing synonyms for this type of approach (angular, periangular, high submandibular approach), mostly referring to the skin incision variants, the authors propose replacing the potentially misleading term transmasseteric anteroparotid approach (TMAP) with transmasseteric infraparotid approach (TMIP) which describes the anatomical transection steps more precisely, thus avoiding the confusion associated with the term anteroparotid approach.

\section{References}

1. Al-Moraissi EA, Ellis E, Neff A. Does encountering the facial nerve during surgical management of mandibular condylar process fractures increase the risk of facial nerve weakness? A systematic review and metaregression analysis. J Cranio-Maxillofac Surg 2018; 46 (8): 1223-1231.

2. Al-Moraissi EA et al. Does the surgical approach for treating mandibular condylar fractures affect the rate of seventh cranial nerve injuries? A systematic review and meta-analysis based on a new classification for surgical approaches. J Cranio-Maxillofacial Surg 2018; 46 (3): 398-412.

3. Li J, Yang H, Han L. Open versus closed treatment for unilateral mandibular extra-capsular condylar fractures: a meta-analysis. J Cranio-Maxillofacial Surg 2019; 47 (7): 1110-1119.

4. Mercuri LG, Steinberg M J. Sequencing of care for multiple maxillofacial injuries. Principles of oral and maxillofacial surgery. Philadelphia: JB Lippincott Co, 1992; 615-622.

5. Eckelt U, Loukota RA. Fractures of the Mandibular Condyle-Approaches and Osteosynthesis. Eberl Medien 2017.

6. Schneider M et al. Open reduction and internal fixation versus closed treatment and mandibulomaxillary fixation of fractures of the mandibular condylar process: a randomized, prospective, multicenter study with special evaluation of fracture level. J Oral Maxillofac Surg 2008; 66 (12): 2537-2544.

7. Zrounba $\mathbf{H}$ et al. Epidemiology and treatment outcome of surgically treated mandibular condyle fractures. A five years retrospective study. J Cranio-Maxillofacial Surg 2014; 42 (6): 879-884.

8. Neff A et al. Position paper from the IBRA symposium on surgery of the head - the 2nd international symposium for condylar fracture osteosynthesis, Marseille, France 2012. J Cranio-Maxillofac Surg 2014; 42 (7): 1234-1249. 
9. Blumer M et al. Outcome of surgically treated fractures of the condylar process by an endoscopic assisted transoral approach. J Oral Maxillofac Surg 2019; 77 (1): 133.e1-133.e9.

10. Rozeboom AVJ et al. Open treatment of condylar fractures via extraoral approaches: A review of complications. J Cranio-Maxillofac Surg 2018; 46 (8): 1232-1240.

11. Güerrissi JO. A transparotid transcutaneous approach for internal rigid fixation in condylar fractures. J Craniofac Surg 2002; 13 (4): 568-571.

12. Schoen R et al. Preliminary functional results of endoscope-assisted transoral treatment of displaced bilateral condylar mandible fractures. Internat J Oral Maxillofac Surg 2008; 37 (2): 111-116.

13. Imai $T$ et al. Surgical approaches for condylar fractures related to facial nerve injury: deep versus superficial dissection. Internat J Oral Maxillofac Surg 2019; 48 (9): 1227-1234.

14. Li $\mathbf{Z}$ et al. Modified tragus edge approach for mid-level or low condylar fractures. Internat J Oral Maxillofac Surg 2016; 45.9): 1100-1103.

15. Meyer $\mathbf{C}$ et al. Clinical experience with osteosynthesis of subcondylar fractures of the mandible using TCP plates. J Cranio-Maxillofac Surg 2008; 36 (5): 260-268.
16. Parihar VS et al. Retromandibular transparotid approach compared with transmasseteric anterior parotid approach for the management of fractures of the mandibular condylar process): a prospective randomised study. Brit J Oral Maxillofac Surg 2019; 57 (9): 880-885.

17. Pau M et al. Use of a modified high submandibular approach to treat condylar base fractures: experience with 44 consecutive cases treated in a single institution. J Cranio-Maxillofac Surg 2016; 44 (10): 1641-1645.

18. Rozeboom AVJ et al. Clinical outcomes in the treatment of unilateral condylar fractures: a cross-sectional study. Internat J Oral Maxillofac Surg 2018; 47 (9): 1132-1137.

19. Trost O, Trouilloud P, Malka G. Open reduction and internal fixation of low subcondylar fractures of mandible through high cervical transmasseteric anteroparotid approach. Brit J Oral Maxillofac Surg 2009; 67 (11): 2446-2451.

20. Wilson AW, Ethunandan M. Brennan PA. Transmasseteric anteroparotid approach for open reduction and internal fixation of condylar fractures. Brit J Oral Maxillofac Surg 2005; 43 (1): 57-60.

21. Boehle AP et al. Transoral vs. extraoral approach in the treatment of condylar neck fractures. . J Cranio-Maxillofac Surg 2015; 43 (2): 224-231.

Received October 27, 2020. Accepted December 5, 2020. 